\section{Result}

Before and after (Figures 6 and 7) pictures show the result of 2,349 grafts (4,361 hairs) after 12 months.

\section{Female Hairline Extension vs. Hairline Advancement}

1. Speed: Hairline advancement can change the appearance within a couple of weeks instead of months.

2. Naturalness: Unless combined with follicular unit transplantation, hairline advancement cannot achieve the naturalness of hairline extension.

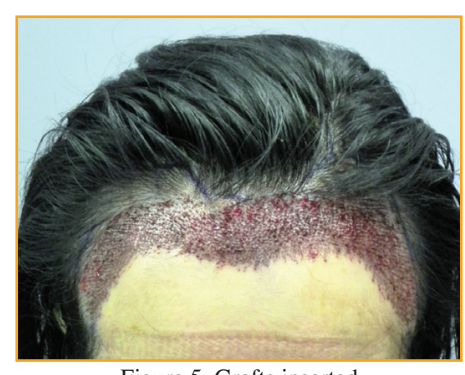

Figure 5. Grafts inserted

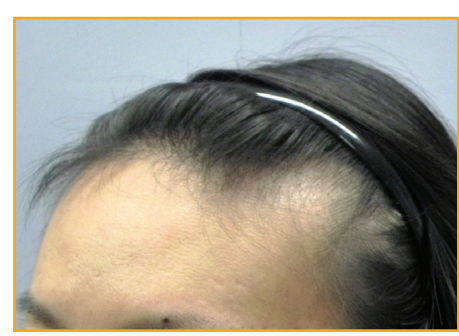

Figure 6. Before surgery

\section{Unisex Hairline}

More men are actually seeking a softer hairline with a rounded apex. The hairline extension approach can also be applied to them.

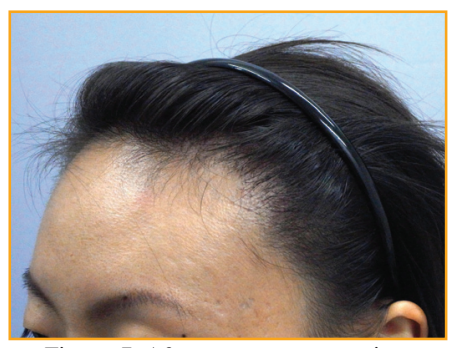

Figure 7. After surgery, same patient

\title{
Integrating follicular unit extraction into a hair restoration office
}

\author{
James A. Harris, MD, and Tina Lardner Greenwood Village, Colorado, USA jaharris@hsccolorado.com
}

Below is a description by Tina of how I have integrated FUE into my practice. Not only has there been a learning curve for myself but my staff has also had to modify their working routine. -James Harris

When Dr. James Harris decided to integrate FUE into his practice in 2003, he was faced with some challenges, such as the time commitment necessary to conquer the learning curve, integrating a new procedure into the clinic workflow, and maintaining the high-quality grafts that he expected. For the surgical assistants, some felt that with FUE in general, their role in the operating room would be eliminated because the grafts are already dissected.

The challenges that were initially faced were eventually overcome and over the past 10 years the clinic has developed a workflow that incorporates both strip surgeries and FUE, therefore giving patients more surgical options. The workload once carried by the surgical assistants to generate grafts has now shifted to the physician. The role of the surgical assistant, although different, is just as critical in the success of the surgical outcome.

\section{Physician Experience}

It can take six months to a year for a surgical assistant to develop the coordination and skill necessary to efficiently perform the tasks involved with cutting and placing grafts, and learning how to perform FUE is no different. There also is a learning curve that the physician must overcome to be proficient; therefore, it is important to start with smaller, 300-500 graft surgeries. Once the physician develops an efficient technique with minimal transection, the surgeries can be larger.

\section{Scheduling Staff}

The speed of extraction and the size of the surgery will dictate the staff needed to perform a FUE surgery. For instance, a smaller FUE surgery of 500 grafts or less and/or a physician with limited experience should have two assistants to assist, inspect, count, and place grafts. For an experienced physician performing larger surgeries of 1,500 grafts or more, three to four assistants are needed. It's important to keep graft counts up-todate so the physician can determine how many grafts to extract from a given area for even distribution.

\section{Scheduling Multiple Surgeries}

Determining the time it takes for a physician to perform a strip and FUE surgery and the capabilities of the staff will help determine how to stagger two surgeries in one day. Once the time is figured out to do both types of surgery, look for downtime during each surgery where the physician can see the other patient. For instance, for a strip surgery, some downtime may be the time after the strip is removed and the patient is being prepped for site making. Since FUE requires more physician time, a strip surgery is typically scheduled first, followed by the FUE surgery. As grafts are generated, one assistant from the strip surgery can move to the FUE surgery and begin graft inspection, sorting, and counting. When the inspecting assistant is unable to keep up with graft production, another assistant comes in from the other surgery to help out.

\section{Role of the Surgical Assistant}

There are critical duties that range from patient preparation, graft inspection, and graft placement to post-operative care that require the surgical assistant's active participation throughout the surgery. Below details a typical day during an FUE surgery and the role of the surgical assistants.

\section{Patient Preparation}

Once the patient arrives, consent forms are signed, photos are taken, and the surgical plan is discussed with the physician. The patient is escorted to the operating room where the donor area is shaved and anesthetized. Proper hair length should be $1-1.5 \mathrm{~mm}$ in length. If the hair is too long, it is difficult for the physician to $\Rightarrow$ page 128 
How I Do It

$\checkmark$ from page 127

thread the hair into the punch. If it's too short, it is difficult to determine the hair emergence angles. Instead of shaving the entire donor area, another option is an individual hair shave technique where follicular units are shaved one at a time.

For scalp anesthesia, the donor area is marked by the physician using a surgical marker. The recipient area is connected to the donor area by numbing the anterior temple, above the ear, and medially just below the occiput (Figure 1). Another line of anesthetic is required to anesthetize the greater occipital nerves (Figure 2).

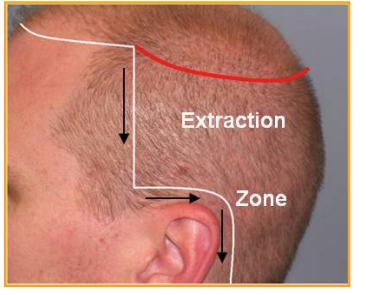

Figure 1. The recipient area is connected to the donor area.

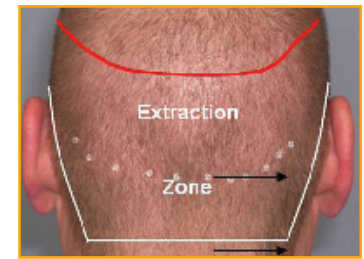

Figure 2. The greater occipital nerves are anesthetized.

\section{Assisting in Surgery}

After the patient is anesthetized, the patient is positioned for graft dissection. The patient is placed in the prone position (some physicians may prefer a seated position for the patient) to give the physician access to the occipital area and then later on his or her side to access the temples. It is the technician's responsibility to adjust lighting, notify the physician of transections and capping, maintain hemostasis, and keep out of the way of the physician.

When extracting grafts, a single forceps is used to lift the graft in the direction it exits the skin (Figure 3). If tethering is noted, a second forceps is applied for a "hand-over-hand" technique (Figure 4). The teeth at the tip of the forceps should never interlock when pulling grafts out due to the risk of crush injury to the follicles.

Capping can occur while extracting grafts from the skin and it tends to be most common with mushy dermis and scar tissue where there is increased tethering. As the graft is extracted, the epidermis slides off and the follicles are left behind. Sometimes capped grafts can be removed by using the forceps to pull the hair up until the graft lifts up slightly (Figure 5), and then another forceps is used to grasp the graft and pull it out of the skin (Figure 6). If the graft doesn't lift up when the hair is pulled, it is left in place where the epidermis will grow over the capped area.

\section{Graft Inspection}

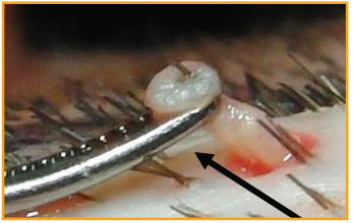

Figure 3. The graft is lifted in the direction it exits the skin.

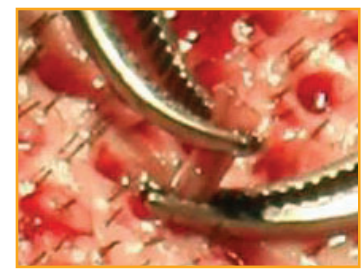

Figure 4. The "hand-over-hand" technique.

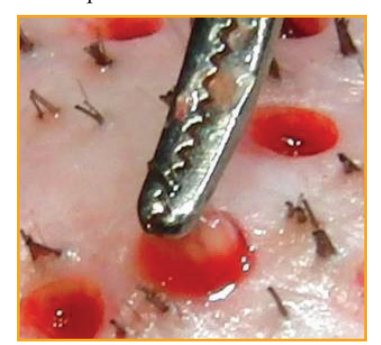

Figure 5. Forceps pulls the hair up.

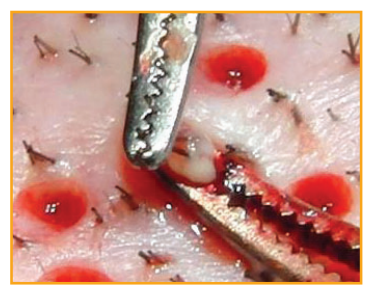

Figure 6. Another forceps is used to grasp the graft and pull it out of the skin

As grafts are extracted, one or two assistants are needed to inspect, count, and sort them. An FUE graft contains a round epidermal cap, the follicles, and limited surrounding tissue (Figure 7). Transections are categorized into three groups: cut follicles, stripped sheath, and damaged follicle bulb (Figure 8). When comparing telogen with transected follicles, the telogen follicles have thick hair shafts with bulbs that are blurred and close to the surface, whereas transected follicles have a distinct cut (straight line) where it was transected (Figure 9). In our office, no trimming of the epidermis is done, however, we do remove any hair fragments and excess fat globules. The epidermal cap is left on as

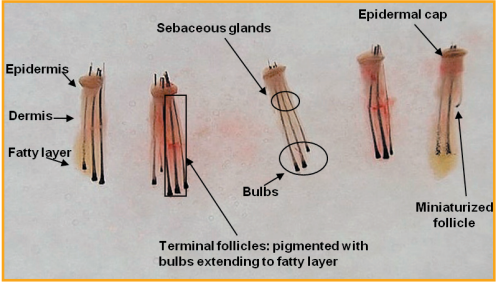
Figure 7. FUE graft anatomy.

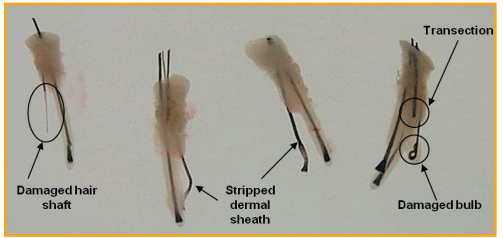

Figure 8. Transection groups. it aids in graft placement. Since the follicles are left behind in the head, caps are counted as a cap, not as a transection, and discarded.

\section{Graft Placement}

Since FUE grafts tend to be more delicate than strip grafts, they are more

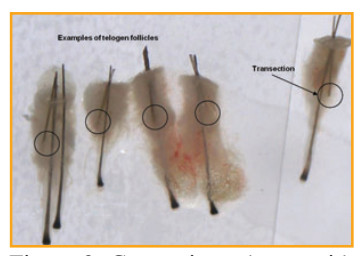

Figure 9. Comparing telogen with transected follicles. prone to injury and desiccation. An experienced staff trained in graft placement is recommended to place FUE grafts when using traditional forceps placement because they generally have the "touch" that inexperienced placers lack. New insertion techniques using Choi implanter pens, or something similar in pre-made sites made be the physician, limit graft manipulation and can be used by inexperienced placers.

Special considerations should be observed when placing FUE grafts. Grafts need to be completely submerged in saline when loaded onto the finger to be placed. Limit the time on the finger to no more than three minutes because the grafts will begin to dry out or get too warm.

Ideally, grafts should be grasped at the fat and inserted into the site. Since FUE grafts have limited tissue surrounding the follicles, they can form "hanging" follicles that are splayed (spread apart) and/or bulbs of different lengths (Figure 10). This can pose a challenge when placing grafts and special attention must be made when grasping the graft. Individual follicles in multi-haired grafts are difficult to grasp in a bundle formation; they tend to flatten out in a "picket fence" configuration making placement difficult. Grasping the graft too firmly can easily crush delicate bulbs. Grasping too high can cause the follicles to bend when inserted (Figure 11). This could cause hair fragment retention (Figure 12) and should be removed if the hairs are not expelled during the post-op period. Grasping splayed, uneven bulbs can be somewhat tricky and, if

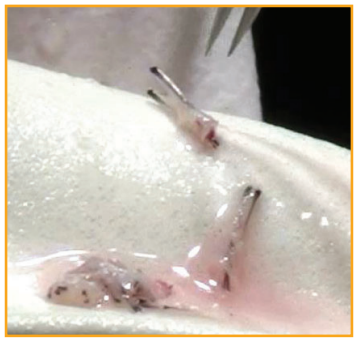

Figure 10. Limited surrounding tissue can give bulbs a splayed (spread apart) appearance. done incorrectly, can cause the upper follicle to bend (Figure 13). Carefully grasp the lower bulbs and insert to the point where the next bulb is at, then insert the next bulb until the graft's epidermal cap is level with the skin (Figure 14). 


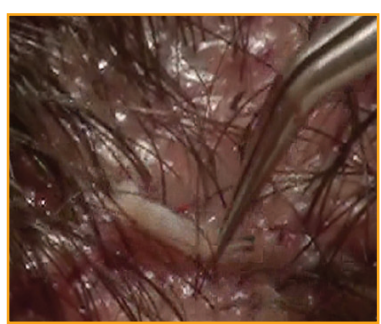

Figure 11. Grasping too high can cause the follicles to bend when inserted.

Since the hair is trimmed short before extraction, determining the curl of the hair is sometimes difficult. Hair orientation is critical to placing grafts, especially in areas like the temple and crown where angles are more acute. The epidermal cap is left on because it dictates the orientation of the hair. If the cap surface is not even with the skin, the hair will not point in the correct direction (Figure 15).

\section{Post-operative Care}

It is normal to have slight oozing and scabbing in the extraction area after surgery. Try to limit cleaning the extraction area with hydrogen peroxide; removing clots will cause the sites to bleed. If the patient is totally shaved, Telfa is applied to the donor area after surgery and is left on overnight (Figure 16). With FUE surgery, most patients find post-operative pain to be significantly less than strip harvesting. Patients are given a sleeper, an over-thecounter pain medication, and a narcotic if needed, and they are instructed to wash their hair the next day as they would for a strip surgery.

\section{Conclusion}

I have found that a team approach by the physician and assistant has contributed to the success of our surgeries. Cross-training

Figure 13. If not grasped correctly, upper follicle to bend.

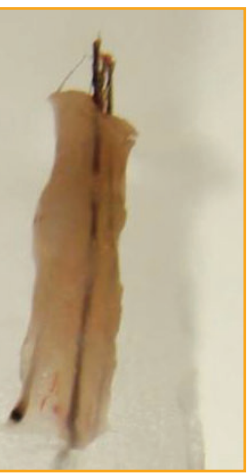
Figure 14. Insert until the graft's
epidermal cap is level with the skin. epidermal cap is level with the skin.

staff to do all aspects of strip surgery (slivers, graft cutting and placing) and FUE surgery gives the office more flexibility when performing two surgeries. Hair restoration diversity not only gives assistants a broader knowledge base and new skill set used to advance the field of hair restoration surgery, but it also gives patients another surgical option. Quality care and attention to detail are what make the "revised" role of the assistant important in the outcome of FUE surgery. Whether it is strip harvesting or FUE, the role of the assistant remains an integral part of hair restoration.

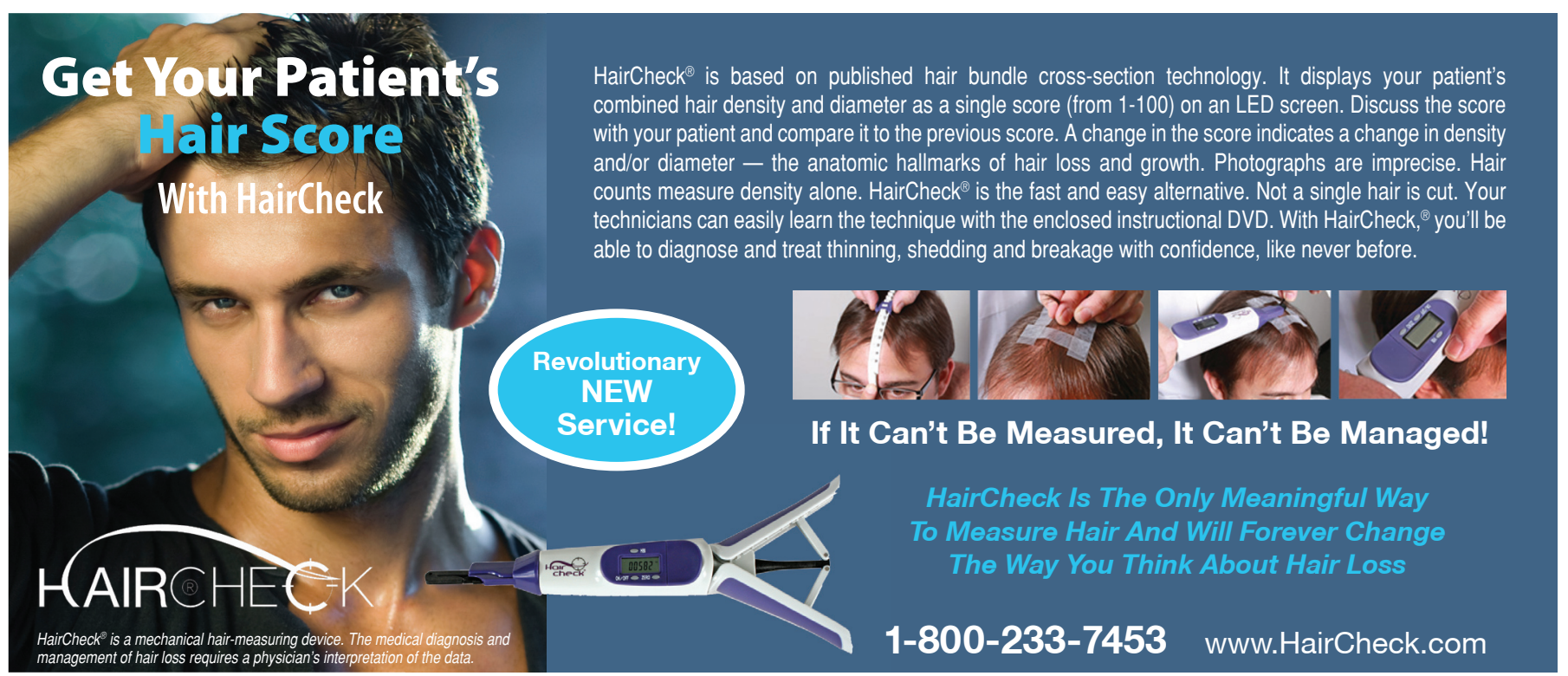

www.ISHRS.org 